\title{
On the linear stability of sheared and magnetized jets without current sheets - relativistic case
}

\author{
Jinho Kim, ${ }^{1,2 \star}$ Dinshaw S. Balsara, ${ }^{1 \star}{ }^{\text {Maxim }}$ Lyutikov $^{3}$ and Serguei S. Komissarov ${ }^{4}$ \\ ${ }^{1}$ Physics Department, College of Science, University of Notre Dame, 225 Nieuwland Science Hall, Notre Dame, IN 46556, USA \\ ${ }^{2}$ Korea Astronomy \& Space Science Institute, 776 Daedeokdae-ro, Yuseong-gu, Daejeon 34055, Korea \\ ${ }^{3}$ Department of Physics, Purdue University, 525 Northwestern Avenue, West Lafayette, IN 47907-2036, USA \\ ${ }^{4}$ Department of Applied Mathematics, The University of Leeds, Leeds LS2 9GT, UK
}

Accepted 2017 November 24. Received 2017 November 23; in original form 2017 May 11

\begin{abstract}
In our prior series of papers, we studied the non-relativistic and relativistic linear stability analysis of magnetized jets that do not have current sheets. In this paper, we extend our analysis to relativistic jets with a velocity shear and a similar current sheet free structure. The jets that we study are realistic because we include a velocity shear, a current sheet free magnetic structure, a relativistic velocity and a realistic thermal pressure so as to achieve overall pressure balance in the unperturbed jet. In order to parametrize the velocity shear, we apply a parabolic profile to the jets' 4-velocity. We find that the velocity shear significantly improves the stability of relativistic magnetized jets. This fact is completely consistent with our prior stability analysis of non-relativistic, sheared jets. The velocity shear mainly plays a role in stabilizing the short wavelength unstable modes for the pinch as well as the kink instability modes. In addition, it also stabilizes the long wavelength fundamental pinch instability mode. We also visualize the pressure fluctuations of each unstable mode to provide a better physical understanding of the enhanced stabilization by the velocity shear. Our overall conclusion is that combining velocity shear with a strong and realistic magnetic field makes relativistic jets even more stable.
\end{abstract}

Key words: instabilities - MHD - relativistic processes - methods: numerical-stars: jets galaxies: jets.

\section{INTRODUCTION}

Jets are a common phenomenon in many energetic astrophysical systems, including active galactic nuclei (AGNs; Rees 1978), young stellar objects (YSOs; Reipurth et al. 1998), X-ray binaries and gamma ray bursts (GRBs). There are strong reasons to believe that magnetic fields are crucial for their production, collimation and acceleration. This conclusion is based on numerous theoretical studies (beginning with the pioneering works by Blandford 1976; Lovelace 1976; Blandford \& Znajek 1977; Blandford \& Payne 1982) and simulations (e.g. Komissarov 1999; Koide et al. 2000; Nishikawa et al. 2005; Hawley \& Krolik 2006; McKinney 2006; Komissarov et al. 2007; McKinney \& Narayan 2007; Komissarov \& Barkov 2009; McKinney \& Blandford 2009; Tchekhovskoy, Narayan \& McKinney 2011). The magnetic models are more complex and hence more difficult to analyse compared to those for unmagnetized flows. Moreover, the observations show that for many astrophysical jets the flow speed is very close to the speed of light, with Lorentz factors up to $\gamma_{0}=50$ for the AGN jets (Lister et al. 2009) and up

^E-mail: jkim46@nd.edu (JK); dbalsara@nd.edu (DSB) to $\gamma_{0}=1000$ for the GRB jets (Piran 2004; Rykoff et al. 2009). This makes the problem even more challenging.

Although many features of astrophysical jets are suggestive of instabilities, these flows appear to be significantly more stable compared to their terrestrial and laboratory counterparts. Indeed, they are capable of propagating huge distances, up to $10^{9}$ of their initial radius, without losing integrity. There is no commonly accepted explanation to this remarkable property, and it remains a subject of ongoing debate.

Most analytical stability studies are based on linear stability analysis. There are many examples of such studies with application to astrophysical jets, in both the non-relativistic (e.g. Hardee 1979, 1982; Cohn 1983; Payne \& Cohn 1985; Appl \& Camenzind 1992; Hardee et al. 1992; Appl 1996; Bodo et al. 1996; Begelman 1998; Appl, Lery \& Baty 2000; Bonanno \& Urpin 2011; Kim et al. 2015; Bodo et al. 2016; Kim et al. 2016) and the relativistic (e.g. Istomin \& Pariev 1994, 1996; Lyubarskii 1999; Tomimatsu et al. 2001; Narayan, Li \& Tchekhovskoy 2009; Bodo et al. 2013; Kim et al. 2017) regimes. In order to keep the mathematics simple and tractable, such studies usually make a number of simplifying assumptions about the structure of the jet. To be more specific, the assumptions are tuned in such a way that the linearized boundary 

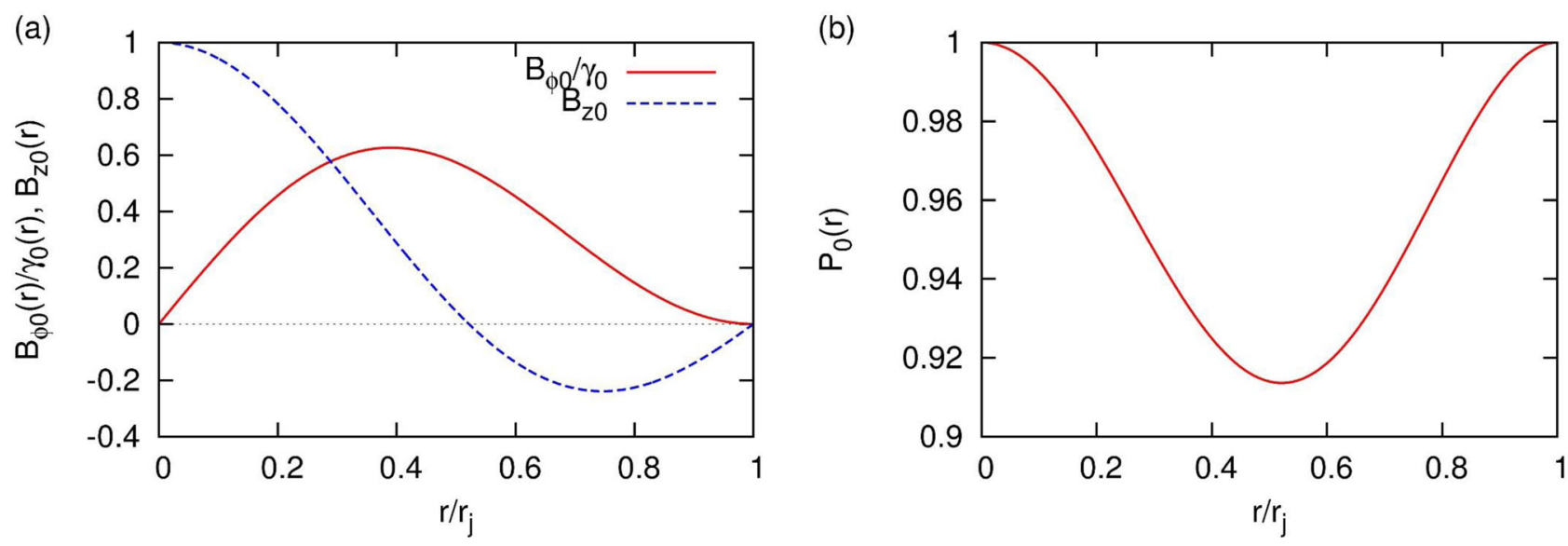

Figure 1. (a) The toroidal magnetic field (red solid line) and the axial field (blue dashed line) as a function of the jet radius from Gourgouliatos et al. (2012). Notice that the toroidal magnetic field is proportional to the Lorentz factor of the jet. The fields are zero at the jet boundary, resulting in jets that do not have a current sheet at the boundary. (b) The corresponding gas pressure in the jet as a function of jet radius.

value problem has eigensolutions which reduce to Bessel functions. However, such simplifications lead to rather artificial flows and it is not entirely clear if the conclusions about the stability of such simple, idealized jets are sufficiently generic. For example, it is normally required to adopt the top-hat velocity profile for the jet (Istomin \& Pariev 1994, 1996; Lyubarskii 1999; Kim et al. 2017) and a very particular simple magnetic fi and a very parIstomin \& Pariev 1994, 1996; Hardee 2007; Narayan, Li \& Tchekhovskoy 2009). Furthermore, not many stability analysis studies of the jet with the internal velocity profile (shear) have been done due to the same reasons [Ferrari, Massaglia \& Trussoni 1982; Perucho, Marti \& Hanasz 2005; Perucho et al. 2007 for the slab jet, Birkinshaw 1991; Urpin 2002; Narayan, Li \& Tchekhovskoy 2009; Nalewajko \& Begelman 2012 for the relativistic astrophysical (cylindrical) jet]. In order to handle more general jet structures, one has to resort to numerical integration of the linearized system. Though the linearized system is much simpler than the original non-linear system it is still too complex to allow standard special functions as its eigensolutions. Such an approach has already been proven and utilized in applications to astrophysical jets (Bodo et al. 2013; Kim et al. 2015, 2016, 2017).

In our first study of this series (Kim et al. 2015), we investigated the stability of non-relativistic jets with a top-hat velocity profile and the non-trivial magnetic field given by Gourgouliatos et al. (2012) which does not have a current sheet at the surface of the jet. We concluded that such a magnetic field promotes reduced growth rates and narrower unstable domains for both pinch and kink modes. In our second work (Kim et al. 2016), we relaxed the standard assumption of the top-hat velocity profile and considered a parabolic profile which introduces velocity shear inside the jets. This leads to further narrowing of the instability windows and reduction of the growth rates. In that work, we also presented a novel way of visualizing the unstable modes based on pressure fluctuations. In our third work (Kim et al. 2017) we extended our study to relativistic jets, both magnetic (as in Gourgouliatos et al. 2012) and unmagnetized, with a top-hat velocity profile. The results show further stabilization due to the relativistic effects.

In this paper, we describe the next step in our study of relativistic jets where we add a velocity shear inside of the jet while preserving the magnetic field structure of Gourgouliatos et al. (2012). All the jets models describe a cylindrical flow which has force balance in the radial direction. Section 2 gives the details of the unperturbed relativistic sheared magnetized jets which we consider in our study. A brief introduction to our method is also documented. Section 3 describes the properties of the fundamental pinch mode and in Section 4 we give the results for the first reflection pinch mode. Section 5 presents a study of the fundamental kink mode and Section 6 focuses on the first reflection kink mode. Discussion and conclusions are presented in Section 7.

\section{DESCRIPTION OF THE UNPERTURBED JET STRUCTURE}

This paper is a sequel to the non-relativistic and relativistic works by Kim et al. (2015, 2016, 2017). Consequently, all the notations in this paper are closely consistent with those in the previous papers.

The unperturbed models of jets in this paper do not have current sheets at the boundary of the jet, nor do the jets require a return current over the length of the jet. Gourgouliatos et al. (2012) present a special configuration of the magnetic field with this property. In this model, the poloidal as well as the toroidal magnetic fields vanish at the surface of the jet so that one avoids the formation of a current sheet at the surface of the jet. The axial and toroidal magnetic fields are expressed using Bessel functions that depend only on radius. Then the gas pressure is obtained by solving the Grad-Shafranov equation based on the proposed magnetic field structure. The resulting pressure and magnetic field are shown as equations 24-26 in Gourgouliatos et al. (2012). For the sake of completeness, we show the structure of the unperturbed jets that are used throughout the paper. Fig. 1(a) shows the poloidal and toroidal magnetic field structure of the unperturbed jets that are used in this paper. The corresponding gas pressure is shown in Fig. 1(b). Note that the toroidal magnetic field is proportional to the Lorentz factor of the jets since Gourgouliatos et al. (2012) assumed that it is measured by an Eulerian observer.

In the course of our prior papers we have found that the presence of a shear in the axial velocity of the jets tremendously enhances the stability of non-relativistic jets (Kim et al. 2016). In this work, we wish to demonstrate that the presence of a shear in the jets' axial velocity also plays a significant stabilizing role for relativistic jets. In order to parametrize the amount of shear in the axial velocity, we use the same parabolic profile as was used in Kim et al. (2016). However, we use an axial 4-velocity $\left(u_{\mathrm{z} 0}=\gamma_{0} v_{\mathrm{z} 0}\right)$ instead of 3 -velocity $\left(v_{\mathrm{z} 0}\right)$ for the parabolic profile to avoid superluminal speed 
(a)

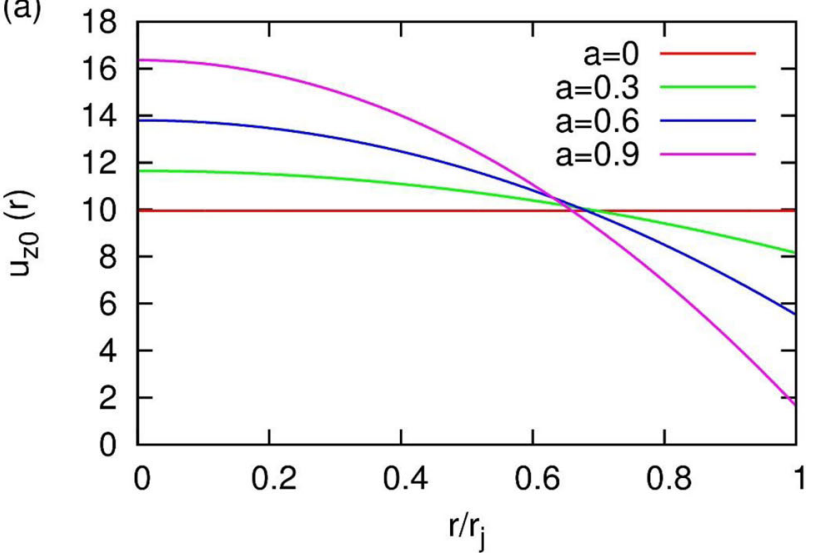

(b)

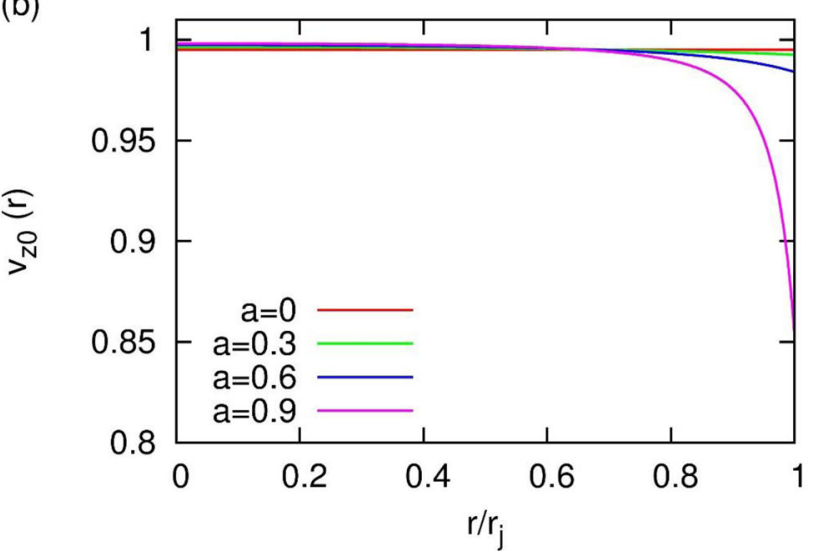

Figure 2. (a) The 4-velocity as a function of jet radius with different strength of velocity shear. It follows the parabolic profile. (b) The corresponding 3-velocity as a function of jet radius.

near the axis. The jets' axial 4-velocity is written as follows:

$u_{z 0}(r)=u_{z 0}^{\max }\left[1-a\left(\frac{r}{r_{j}}\right)^{2}\right]$.

Here, $u_{z 0}^{\max }$ is the maximal on-axis $z$-component of the 4-velocity of the jets. Also, $r_{\mathrm{j}}$ is the jets' radius and is usually set to unity. The parameter, ' $a$ ', ranges from 0 for a top-hat velocity profile $\left(u_{z 0}(r=\right.$ $\left.\left.r_{j}\right)=u_{z 0}(r=0)\right)$ to 0.9 for a jet whose 4 -velocity on the surface is 10 per cent of its on-axis 4-velocity $\left(u_{z 0}\left(r=r_{j}\right) / u_{z 0}(r=0)=0.1\right)$. When parametrizing the shear for a family of jet solutions, we realize that some physical attribute of the jetted flow has to be kept constant. To do this, the maximum on-axis velocities are determined by requiring that the total kinetic energy of the jets stay constant. Here, the jets' relativistic kinetic energy is expressed as

$T=2 \pi \int_{0}^{r_{\mathrm{s}}} \rho_{0} h_{0} \gamma_{0}^{2} v_{z 0}^{2} r \mathrm{~d} r$.

Note that the on-axis velocity of a jet with a high shear has a larger value compared to a mildly sheared jet. In the non-relativistic limit $\left(\gamma_{0} \rightarrow 1, h_{0} \rightarrow 1\right)$, Eq. (2) reduces to the expression of the non-relativistic kinetic energy. Fig. 2(a) shows the 3-velocity profiles considered in this paper with $a=0,0.6,0.9$. The corresponding 3-velocity is easily retrieved with a conversion formula, i.e. $v_{z 0}(r)=u_{z 0}(r) / \sqrt{u_{z 0}^{2}(r)+1}$. This is shown in Fig. 2(b). Our choice of a parabolic profile for the axial 4-velocity gives nearly flat 3velocity profile near the axis and it falls off rapidly at the surface of the jet. Since the parabolic velocity profiles satisfy the Rayleigh criterion (Rayleigh 1896), they are stable. Note that unlike our previous paper (Kim et al. 2016), we do not show the stability of $a=0.3$ jets. This omission is because there is almost no difference in 3-velocity profile between a jet with a top-hat velocity profile and a jet with $a=0.3$. In other words, almost no distinguishable difference in the stability is observed between a jet with $a=0.3$ and a jet with $a=0.0$.

The Mach number is another parameter that characterizes our jet models. The velocity shear gives different Mach numbers at different locations of the unperturbed jet. We, therefore, use integrated quantities for the Mach number. It is defined as follows:

$M=\sqrt{\frac{\int_{0}^{r_{\mathrm{s}}} \rho_{0} h_{0} \gamma_{0}^{2} v_{z 0}^{2} r \mathrm{~d} r}{\Gamma \int_{0}^{r_{s}} P_{0} \gamma_{0}^{2} r \mathrm{~d} r}}=\sqrt{\frac{T}{\pi \Gamma \int_{0}^{r_{\mathrm{s}}} P_{0} \gamma_{0}^{2} r \mathrm{~d} r}}$.
Note that in the non-relativistic limit $\left(\gamma_{0} \rightarrow 1, h_{0} \rightarrow 1\right)$, our present definition of $M$ retrieves the non-relativistic definition of Mach number shown in Kim et al. (2016).

The ratio of the jet and ambient densities, $\eta=\rho_{\mathrm{j}} / \rho_{\mathrm{a}}$ and the plasma beta, $\beta=P_{\mathrm{j} /}\left(B^{2}{ }_{\mathrm{j}} / 2\right)$ are entirely consistent with the previous papers. Here, the subscripts ' $j$ ' and ' $a$ ' represent the jet and ambient medium, respectively. All the jet's physical quantities with subscript ' $\mathrm{j}$ ' are measured in the comoving frame of the fluid.

The specific values of the parameters that are used throughout the paper are as follows: (i) The polytropic exponent, $\Gamma$ is $5 / 3$. (ii) The jet and ambient density ratio, $\eta$, is 0.1 . (iii) The Mach number, ' $M$ ', is 4. (iv) The Lorentz factor of the unperturbed jet, $\gamma_{0}$, is 10 . (v) We use three different values of ' $a$ ' for the unperturbed jet velocity profile. They are $0,0.6,0.9$ from top hat to strong shear profile. (vi) We use three different strengths for the magnetic field. The $\beta$ values are $\infty, 1$ and $1 / 2$ from the non-magnetized jet to the strongly magnetized jet. Note that $\beta$ is only defined on the axis of the jet. Because the $z$-component of the magnetic field is unchanged by a Lorentz transformation, our definition of plasma $\beta$ is equally valid in the rest frame of the jet as in the lab frame.

In Kim et al. $(2015,2016)$ we describe our numerical methods for the linear stability analyses of non-relativistic jets with realistic density, pressure, velocity and magnetic field profiles. We use the same numerical strategy for the analyses of the relativistic jets in Kim et al. (2017). The governing equations for the relativistic extension of our numerical method are detailed in Kim et al. (2017). Moreover, the relativistic linearized equations in Kim et al. (2017) already consider the velocity shear in the jets' profile [see equations 8-14 in Kim et al. (2017)]. The formulation in our previous paper is very general and can be used without modification in this paper. We, therefore, do not repeat all the details of the formulation and the numerical methods in this paper. Note that the perturbation of jets' flow variables has the form $\delta f(t, r, \phi, z)=\delta f(r) \exp (\mathrm{i} \omega t-$ $m \phi-k z)$. We only study the pinch $(m=0)$ and the kink $(m=1)$ modes which are known to be the most destabilizing modes. We only consider the complex number solution of $\omega$ as a function of real $k$. Hence the imaginary part of $\omega$ (i.e., $\omega_{\mathrm{I}}$ ) is the temporal growth rate of the mode.

It is intuitively helpful to understand the jet stability if we set up a stability criterion for the jet. In Kim et al. (2016, 2017) we consider such a criterion of the jets' instability. This criterion is evaluated by comparing the e-folding time due to the instabilities of the jets with the time taken by the perturbation to propagate hundreds of jets' 
radii. The e-folding time of the jet is the inverse of the temporal growth rate, $\tau=1 /\left|\omega_{\mathrm{I}}\right|$. And the time taken by each unstable mode which propagates with its group velocity $\left(v_{\mathrm{g}}\right)$ to reach $\chi$ jet radii is given by $T=\chi r_{\mathrm{j}} / v_{\mathrm{g}}$. We, therefore, say that the instability will not destabilize the jet too much if $\tau \geq T$. This can be written as

$$
\frac{\omega_{\mathrm{I}} r_{\mathrm{j}}}{c_{\mathrm{s}}} \leq \frac{v_{\mathrm{g}}}{\chi c_{\mathrm{s}}} \text {. }
$$

For this paper, we use $\chi=400$. In other words, a jet might be stable if the perturbation of the jet can propagate 400 or more jet radii before one e-folding time. All the figures of the dispersion relations after this point have coloured and dotted lines which represent the thresholds of the stability criterion of each unstable mode. To obtain the group velocity $\left(v_{\mathrm{g}}=\partial \omega_{\mathrm{R}} / \partial k\right)$, we numerically differentiate the real part of $\omega$ with respect to the wavenumber $k$. Note that there is no special physical significance of choosing the value of $\chi=400$. One may increase or decrease $\chi$ value. In this case, the criterion threshold line parallelly moves up and down. In this sense, this stability criterion line is a reference line that can determine whether the perturbation of the jet propagates 400 jet radii in one e-folding time or not. One may interpret jets' stability by comparing with this reference line.

\section{THE STABILITY OF THE FUNDAMENTAL MODE OF THE PINCH INSTABILITY}

Fig. 3 shows the dispersion relation for the fundamental pinch mode instability. Solid and dashed lines are the oscillation frequency $\left(\omega_{R}\right)$ and temporal growth rate $\left(\omega_{\mathrm{I}}\right)$ as a function of wavenumber, respectively. Fig. 3(a) corresponds to non-magnetized jets $(\beta=\infty)$. Fig. 3(b) corresponds to jets with equipartition between the gas and magnetic pressure $(\beta=1)$. Fig. 3(c) is for jets with on-axis magnetic pressure that is twice as strong as the on-axis gas pressure $(\beta=1 / 2)$. Each figure shows three different values of shear, $a=0$ (red), 0.6 (green) and 0.9 (blue). The stability criteria of each unstable mode are shown via dotted lines. Using our thresholds, which are shown by dotted lines in Fig. 3, the jet is very stable regardless the value of the shear parameter ' $a$ ' at all wavelengths that we consider in this paper due to the relativistic effect $\left(\gamma_{0}=10\right)$. The enhanced stability provided by an increasing Lorentz factor has been studied by Kim et al. (2017). Kim et al. (2017) show that the temporal growth rate is inversely proportional to the Lorentz factor square for the fundamental mode of the pinch instability. In Fig. 3, the overall trend is that increasing the shear makes the fundamental mode of the pinch instability more stable for a substantial range of short as well as long wavelengths. This fact is completely consistent with the stability analysis for the non-relativistic sheared jets. See fig. 3 in Kim et al. (2016). From Fig. 3(c) of this paper. which pertains to strongly magnetized jets, we see however that the interaction between strong shear and strong magnetic field does not always cause a monotonic improvement in stability with increasing shear. The same trend is also visible in fig. 3(c) of Kim et al. (2016). It is, nevertheless, heartening to see that the interaction of strong magnetic field and strong shear in Fig. 3(c) of this paper results in a very small range of wavelengths that are only mildly unstable to the fundamental mode of the pinch instability.

We visualize the total pressure perturbation to see physical properties of the unstable modes; this is just as we did for the nonrelativistic sheared jet (Kim et al. 2016). Fig. 4 shows the pressure perturbation in the jet and its ambient medium when the radial displacement of the perturbation at the jet's surface is 20 per cent of jet's radius. We show $5 r_{\mathrm{j}}$ in the radial direction (vertical axis)
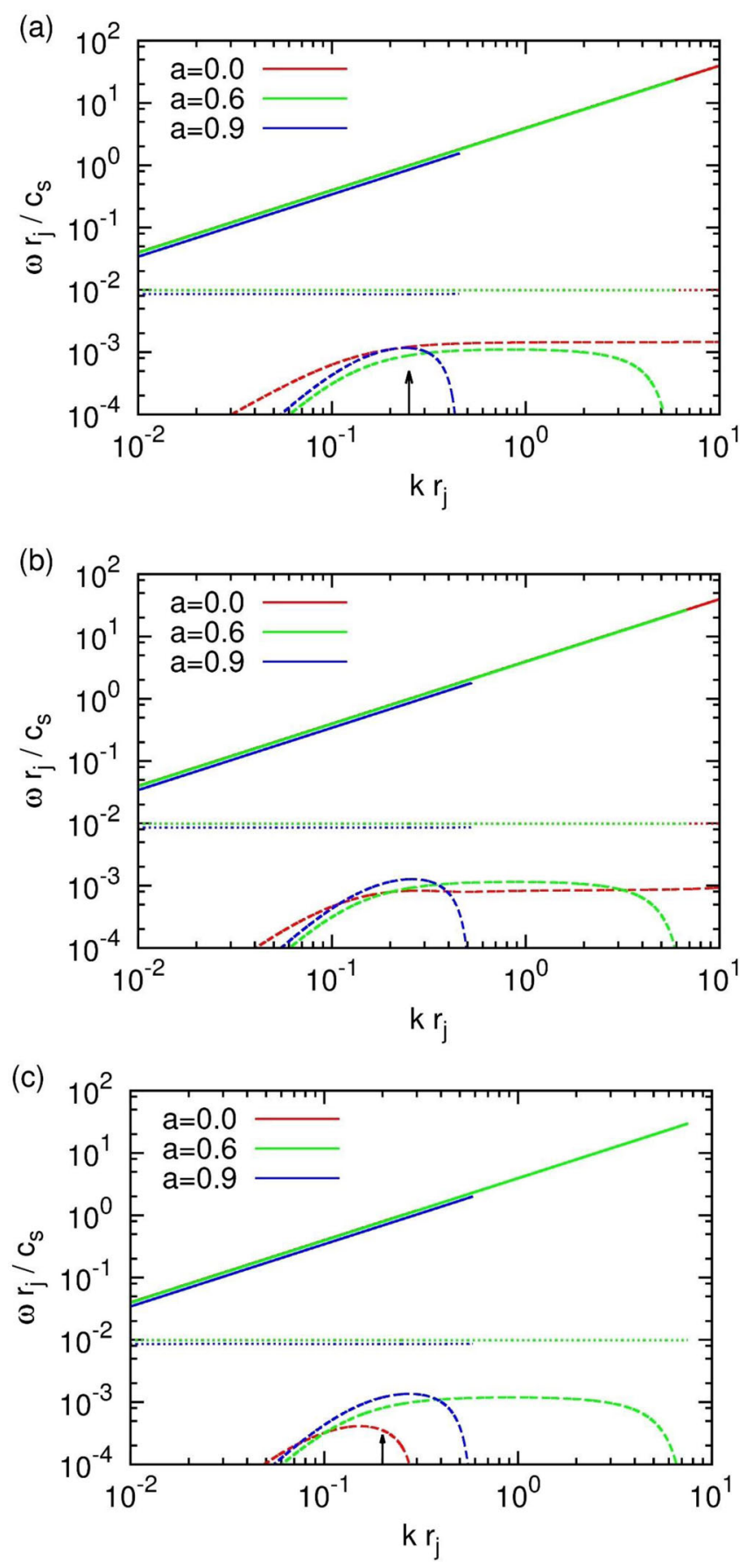

Figure 3. The angular frequency (solid line) and temporal growth rate (dashed line) as a function of longitudinal wavenumber $k$ for pinching $(m=0)$ fundamental mode of (a) a non-magnetized jet, (b) a jet with $\beta=1$ (i.e. magnetic field is in equipartition with gas pressure) and (c) a jet with $\beta=0.5$ (magnetically dominated). The jet has $M=4$ and $\eta=0.1$. Increasing values of the parameter ' $a$ ' indicate increasing shear, with $a=0$ (no shear) to $a=0.9$ (maximal shear). The stability criteria of each unstable mode are shown via dotted lines.

and two wavelengths of the perturbation in $z$-direction (horizontal axis). In Fig. 4, the colours of the pressure perturbation contours match with the colour bar on the right-hand side of each figure. We show the unstable modes with $k r_{\mathrm{j}}=0.25$ that is marked with an arrow in Fig. 3(a). The perturbed boundary surface of the jet (radial 

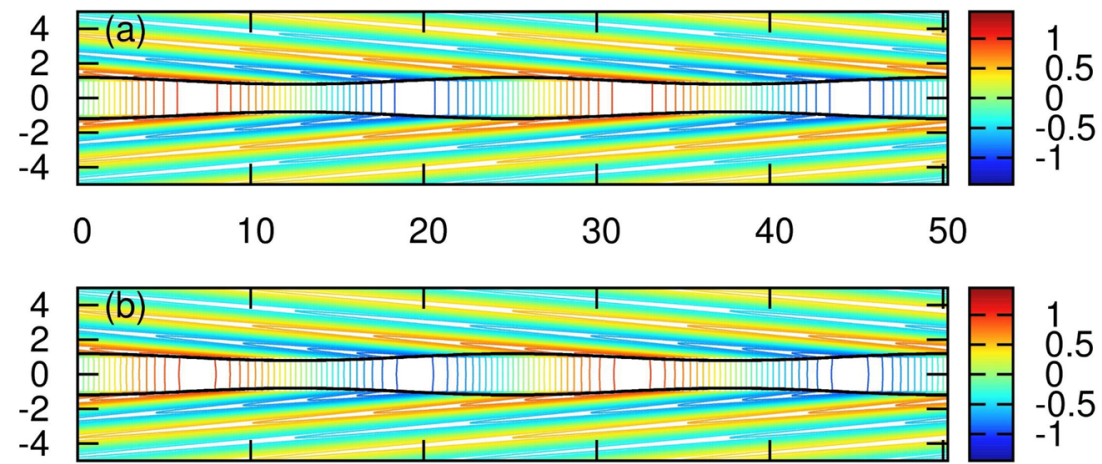

$\begin{array}{llllll}0 & 10 & 20 & 30 & 40 & 50\end{array}$
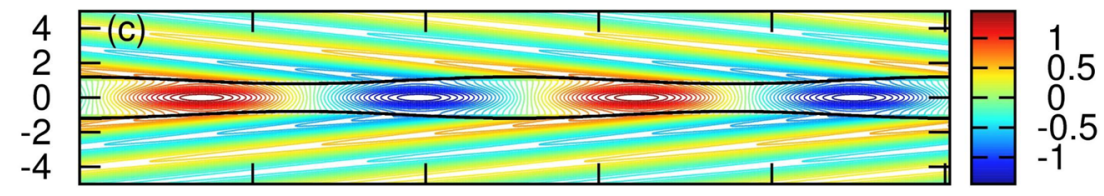

$0 \quad 10$

20

30

40

50

Figure 4. The pressure variation of the fundamental pinch mode in a non-magnetized jet with (a) a top hat profile, (b) $a=0.6$ (moderate shear) and (c) $a=0.9$ (strong shear). For all the cases in Fig. 4 we have $k r_{\mathrm{j}}=0.25$. In all the cases, the jet's boundary has a fluctuation that is 20 per cent of the jet's radius. The pressures are all on the same scale so that the pressures across panels within a figure can be inter-compared.
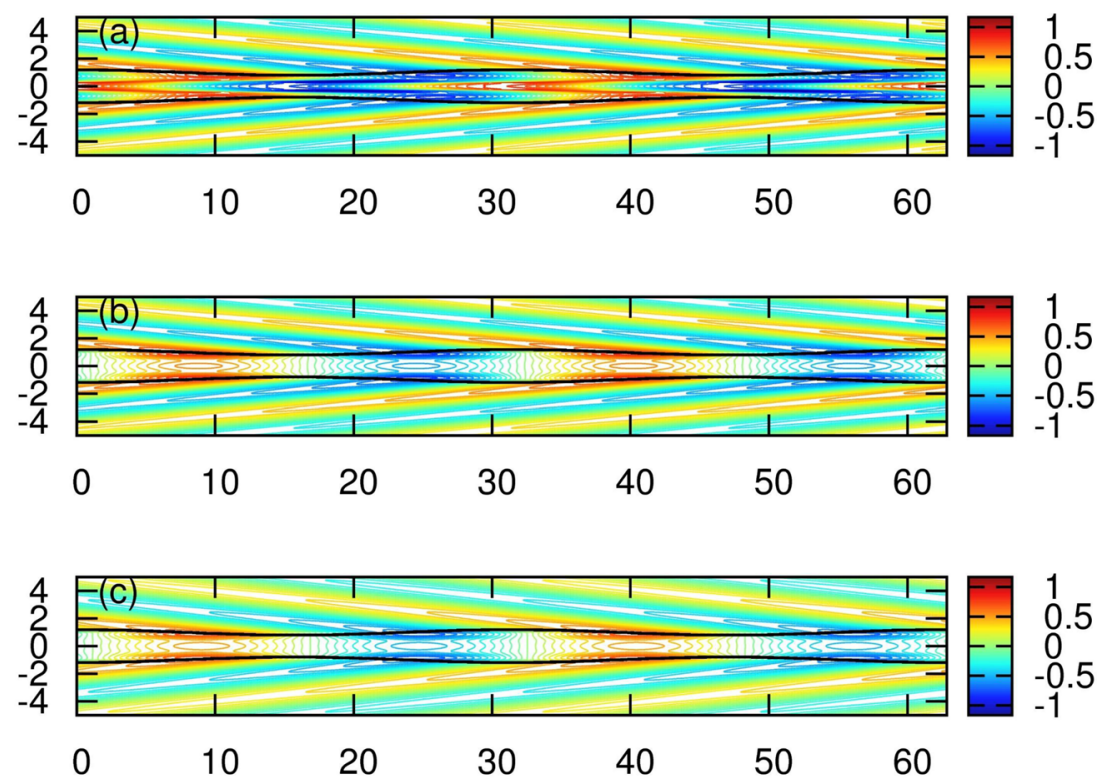

Figure 5. The pressure variation of the fundamental pinch mode in a strongly magnetized jet ( $\beta=1 / 2$ ) with (a) a top hat profile, (b) $a=0.6$ (moderate shear) and (c) $a=0.9$ (strong shear). For all the cases in Fig. 5 we have $k r_{\mathrm{j}}=0.2$. In all the cases, the jet's boundary has a fluctuation that is 20 per cent of the jet's radius. The pressures are all on the same scale so that the pressures across panels within a figure can be inter-compared.

displacement at the surface) is also shown as a black solid line in Fig. 4. Fig. 4(a) shows the pressure for an unmagnetized jet with $a=0$; Fig. 4(b) shows the pressure for an unmagnetized jet with $a=0.6$; Fig. 4(c) shows the pressure for an unmagnetized jet with $a=0.9$. We use the same Mach number, density ratio and perturbed wavelength for all three panels in Fig. 4. The colour scale is also kept fixed for all the panels in Fig. 4 so that the reader can inter-compare across the panels within the figure.

In Fig. 4(a) we can clearly see the longitudinal oscillation within the jet's channel. This property characterizes the fundamental pinch mode of the jet. As the velocity shear increases, the pressure fluctuation inside jet's channel is concentrated on the axis of the jet. Please compare Fig. 4(a) with Fig. 4(c). As a result, the boundary of the jet's channel experiences smaller perturbations. Such a trend is completely consistent with our non-relativistic study in Kim et al. (2016). In other words, the energy loss that occurs from the boundary of the jet to its ambient medium is smaller for a sheared jet than for a jet with a top-hat velocity profile. This gives us a physical appreciation for the role of shear in stabilizing relativistic jets.

Let us focus on the pressure perturbation outside of the jet. It is described as the Mach line of the outgoing sound wave. When we 
write $\theta_{\mathrm{o}}$ as a propagation angle of the outgoing wave at the surface of the jet, the relation between $\theta_{\mathrm{o}}$ and phase velocity $\left(v_{\mathrm{ph}}=\omega_{\mathrm{R}} / k\right)$ is

$\theta_{\mathrm{o}}=\sin ^{-1}\left(\frac{c_{\mathrm{s}, \mathrm{a}}}{v_{\mathrm{ph}}}\right)$,

where $c_{\mathrm{s}, \mathrm{a}}$ is the sound speed of the ambient medium. In Fig. 3, the phase velocity has an almost constant value as the shear in the velocity increases; i.e. the real value of $\omega$ does not change much when the value of ' $a$ ' increases. For this reason, the Mach angle in this paper does not depend much on the strength of the velocity shear, ' $a$ ' if we choose the same wavenumber $k$. Note that the pressure fluctuation figures of the $a=0.9$ jets in our non-relativistic analysis (Kim et al. 2016) have noticeable difference in the Mach angle compared with other jets having smaller velocity shears. This is because the phase velocities of the $a=0,0.3$ and 0.6 jets are much larger than those for the $a=0.9$ jets (see figs 3 and 10 of Kim et al. 2016). In this paper, this situation however does not happen. The phase velocities in Fig. 3 are almost identical.

Fig. 3(c) shows the dispersion relation for the fundamental modes of the pinch instability when the jet is strongly magnetized. By comparing the $a=0$ jet with the $a=0.6$ and 0.9 jets, it is shown that the presence of shear enhances stability at short as well as long wavelengths. Inter-comparison of Fig. 3(a) and Fig. 3(c) shows that the magnetic field only stabilizes the jet at short wavelength. The inclusion of velocity shear is only able to stabilize the long wavelength instability modes and these modes are thought to play an important role in the disruption of the jet.

Fig. 5 is analogous to Fig. 4 but for the magnetically dominated jet $(\beta=1 / 2)$. The wavenumber that we choose is $k r_{\mathrm{j}}=0.2$ which is marked by an arrow in Fig. 3(c). The pressure fluctuation for the $a=0$ jet in Fig. 5(a) is not purely longitudinal any more. Please compare Fig. 5(a) to Fig. 4(a) to see this. Fig. 5(a) shows that the pressure fluctuations are confined closer to the axis of the jet compared to Fig. 4(a). This is because of the effect of the toroidal magnetic field shown in Fig. 1(a). This component of magnetic field can collimate the pressure fluctuations so that they are concentrated nearer to the axis of the jet. Thus, just like the effects by the velocity shear in Fig. 3, magnetized jets have better stability. As we increase the strength of the velocity shear in the magnetized jet, a similar concentration of the pressure fluctuations to the axis of the jet channel occurs in Fig. 5. In both Figs 4 and 5 we observe that the shear breaks down the coherency of the growing modes, therefore contributing to an improved stability.

\section{THE STABILITY OF THE FIRST REFLECTION MODE OF THE PINCH INSTAB I L I T Y}

Fig. 6 shows dispersion relation analogous to Fig. 3 for the first reflection mode of the pinch instability. In Fig. 6, only a narrow range of wavelengths produce unstable modes that are above the stability criterion. From Fig. 6(a), increasing the shear in the velocity results in an improved stability for the first reflection mode at short wavelengths. This fact is valid for the magnetized jets in Figs 6(b) and (c). Especially, the jet with $a=0.9$ shows the onset of the mode mixing with the second reflection mode. Increasing the magnetic field makes the marginal stability point where both real and imaginary parts of $\omega$ vanish shift to shorter wavelengths. However, this effect is not significant. Note that both the growth rate at resonance (maximum growth rate) and the wavenumber at the marginal stability point of the first reflection mode of the pinch
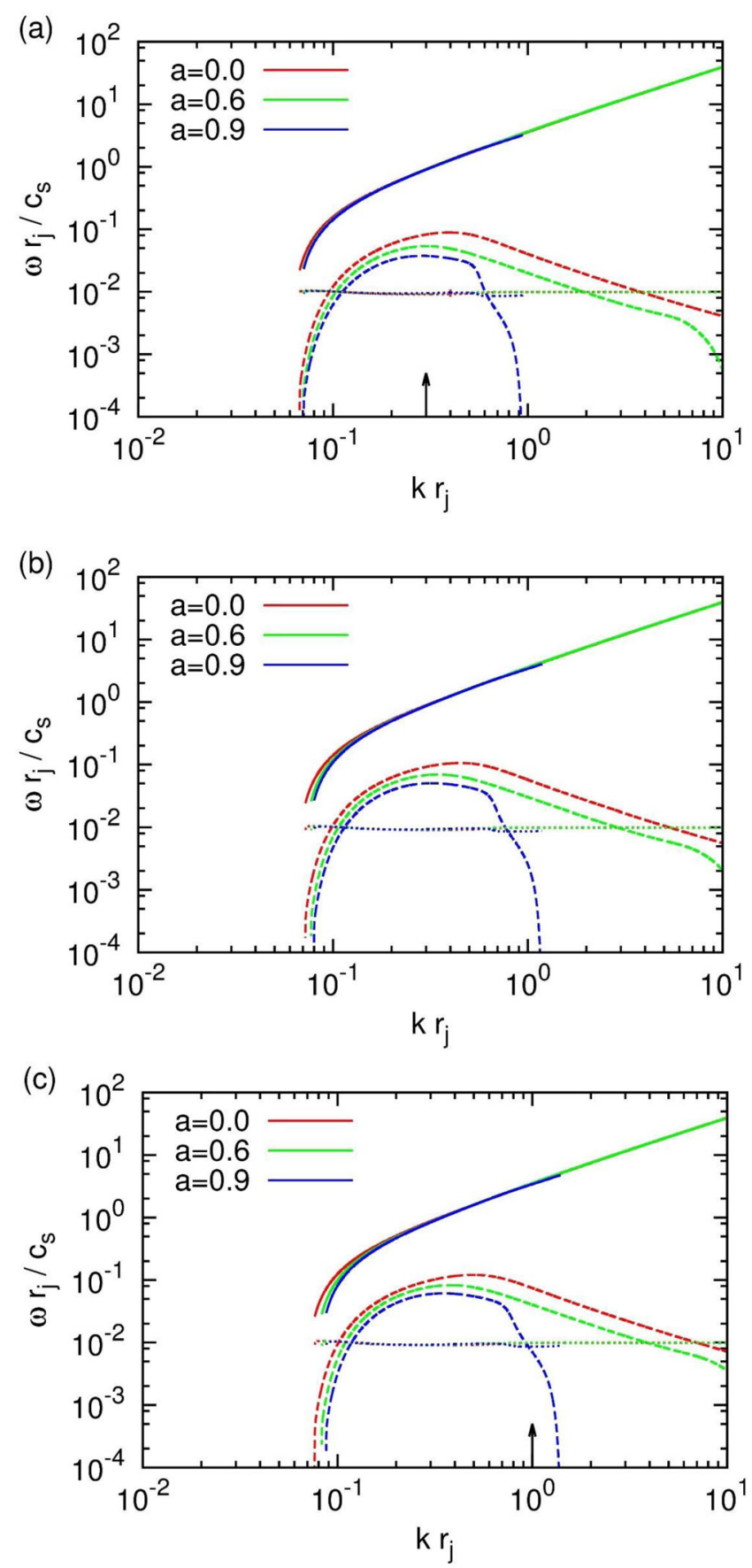

Figure 6. The angular frequency (solid line) and temporal growth rate (dashed line) as a function of longitudinal wavenumber $k$ for pinching $(m=0)$ first reflection mode of (a) a non-magnetized jet, (b) a jet with $\beta=1$ (i.e. magnetic field is in equipartition with gas pressure) and (c) a jet with $\beta=0.5$ (magnetically dominated). The jet has $M=4$ and $\eta=0.1$. Increasing values of the parameter 'a' indicate increasing shear, with $a=0$ (no shear) to $a=0.9$ (maximal shear).

instability is inversely proportional to the Lorentz factor. See Kim et al. (2017) for details.

Fig. 7 shows pressure fluctuation of the unstable first reflection mode of the pinch instability in a non-magnetized jet. The wavenumber that we choose is $k r_{j}=0.3$ which is marked in Fig. 6(a) by an arrow. Figs 7(a)-(c) show the pressure fluctuation for a nonmagnetized jet with $a=0,0.6,0.9$, respectively. It clearly shows 

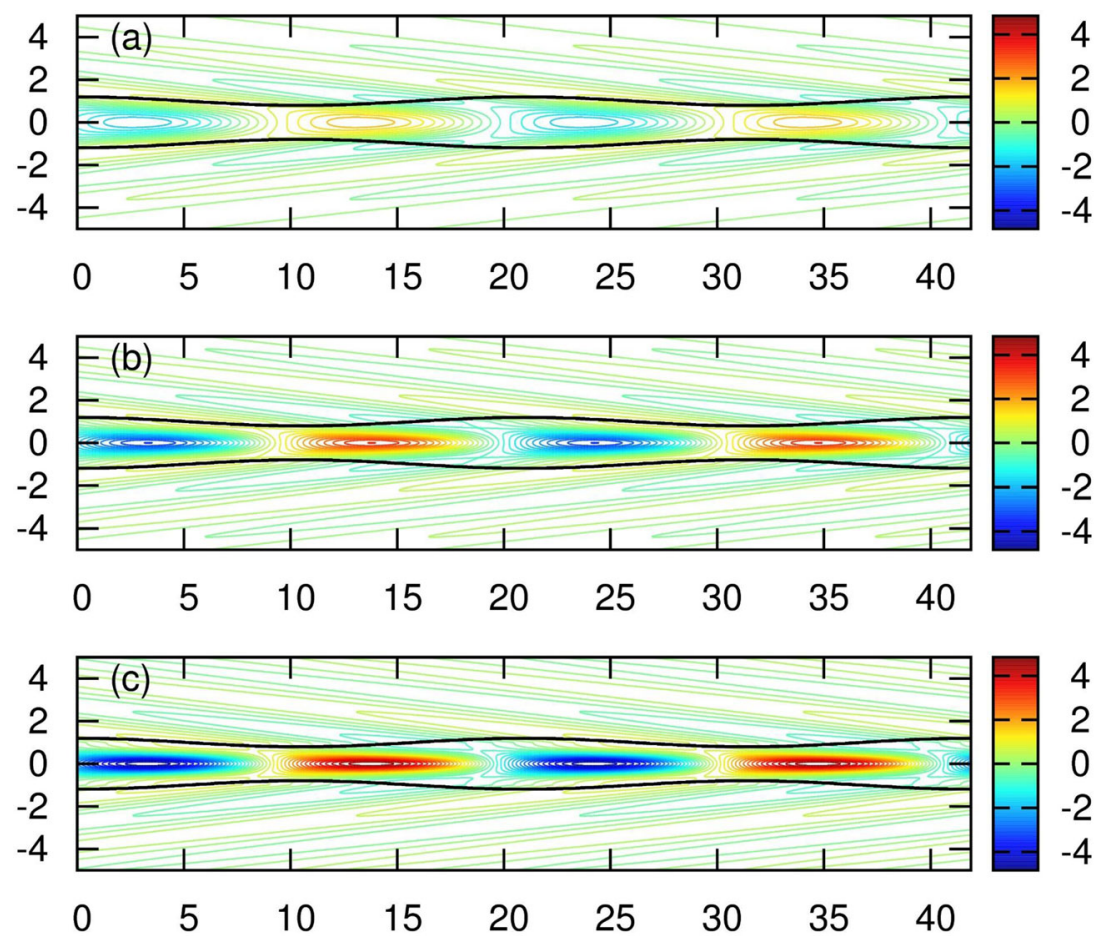

Figure 7. The pressure variation of the first reflection pinch mode in a non-magnetized jet with (a) a top hat profile, (b) $a=0.6$ (moderate shear) and (c) $a=0.9$ (strong shear). For all the cases in Fig. 7 we have $k r_{\mathrm{j}}=0.25$. In all the cases, the jet's boundary has a fluctuation that is 20 per cent of the jet's radius. The pressures are all on the same scale so that the pressures across panels within a figure can be inter-compared.

transverse wave patterns in the reflection mode (cf. the fundamental mode shows the longitudinal wave pattern in Fig. 4a). As shown in Fig. 4, increasing shear causes the pressure fluctuations in the jet to be concentrated on the axis of the jet. The velocity profile with $a=0.9$ in the 4-velocity effectively gives $a=0.2$ in the 3 -velocity. Accordingly, it does not produce a significant difference in the amplitude of sound wave fluctuations at the jet-ambient boundary as shown in the non-relativistic study by Kim et al. (2016). This implies that, specifically for the first reflection modes of the pinch instability, there is no dramatic decrease of the energy loss though the boundary of the jet when the velocity shear increases.

The pressure fluctuations in the first reflection modes of the pinch instability for the strongly magnetized jets are shown in Fig. 8. The wavenumber that we choose here is $k r_{\mathrm{j}}=1$. The confinement of the pressure perturbation close to the axis of the jet in the nonmagnetized jet also appears in the strongly magnetized jet.

In equation (5), we define the propagation angle of the outgoing wave. For the propagation angle of the inside jet, we use the same definition of the propagation angle as in Payne \& Cohn (1985). Their solution of the pressure perturbation is given as

$\delta P(r, z, \phi, t) \sim F_{\mathrm{m}}(\beta k r) \mathrm{e}^{\mathrm{i} \omega t-\mathrm{i} k z-\mathrm{i} m \phi}$,

where the function $F_{\mathrm{m}}$ can be either Bessel function $J_{\mathrm{m}}$ or Hankel function $H_{\mathrm{m}}$. The Bessel function basically has periodicity. Therefore, it is possible to define the effective wave propagation vector for the mode as

$\boldsymbol{k}_{\mathrm{eff}}=k(\hat{z}+\beta \hat{\boldsymbol{r}})$,

where $\hat{z}$ and $\hat{\boldsymbol{r}}$ are the unit vectors in $z$ and $r$ direction. Then the propagation angle of the wave to the normal vector of the surface of the jet can be written as

$\theta=\cot ^{-1} \beta$.
The wave propagation angle of the non-magnetized jets in our analysis exactly follows the above equation. Hence, in Figs 4(a) and 7(a), we obtain the exactly consistent results with the Figs 6 and 7 in Payne \& Cohn (1985). On the other hand, the wave propagation angle of the magnetized jets does not follow the equations (6) and (7) since the pressure perturbation inside the jet's channel does not appear as the Bessel function solution. Although we cannot obtain the propagation angle from equations (6) and (7), we can roughly estimate it from the pressure perturbation figures. Let us try to focus on the Figs 7 and 8 which are the pressure fluctuations of the first reflection modes. From the pattern of the pressure fluctuation inside jet channel, we can estimate the wavelength in $r$ as well as $z$ direction denoted by $\lambda_{r}$ and $\lambda_{z}$, respectively. Since the wavelengths are inverse of wavenumber, the propagation angel would be

$\theta=\tan ^{-1}\left(\frac{\lambda_{r}}{\lambda_{z}}\right)$

The internal wave propagation angle of Fig. 7(a) has a larger value compared with 7(c) because the $r$-directional wavelength $\left(\lambda_{r}\right)$ in Fig. 7(a) is larger than that in Fig. 7(c) while the $z$-directional wavelength $\left(\lambda_{z}\right)$ remain constant at a specific value. This trend is also shown in Fig. 8.

Payne \& Cohn (1985) claim that the growth rate of an unstable mode at short and intermediate wavelengths is large when the reflection coefficient $R$ is large. Here $R$ can be expressed in terms of the propagation angle of the wave to the normal vector of the surface of the jet $\left(\theta_{\mathrm{i}}, \theta_{\mathrm{o}}\right)$ :

$R=\frac{\cot \left(\theta_{\mathrm{i}}+\theta_{\mathrm{o}}\right)}{\cot \left(\theta_{\mathrm{i}}-\theta_{\mathrm{o}}\right)}$

where the subscripts ' $i$ ' and 'o' represent wave propagation angles inside and outside the jet, respectively. In other words, the growth 

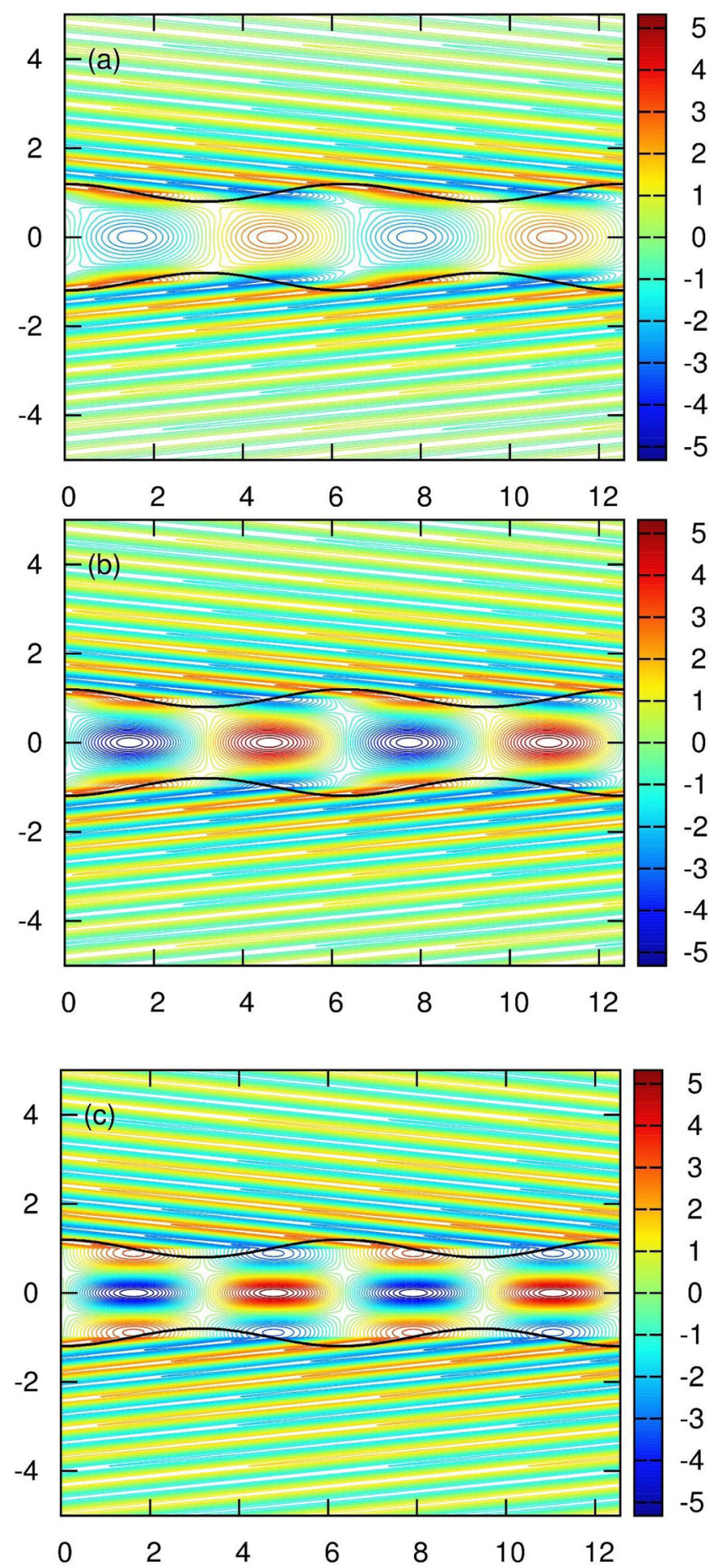

Figure 8. The pressure variation of the first reflection pinch mode in a strongly magnetized jet ( $\beta=1 / 2)$ with (a) a top hat profile, (b) $a=0.6$ (moderate shear) and (c) $a=0.9$ (strong shear). For all the cases in Fig. 8 we have $k r_{\mathrm{j}}=1$. In all the cases, the jet's boundary has a fluctuation that is 20 per cent of the jet's radius. The pressures are all on the same scale so that the pressures across panels within a figure can be inter-compared.

rate has the maximum value when the inside and outside propagation angles are same. In that sense, both angles are almost same in Fig. 8(a) since we choose the wavenumber that is close to the resonance, whereas they are quite different in Fig. 8(c) which is away from the resonant point. (Please see Fig. $8 \mathrm{a}$ and $\mathrm{b}$ closely to see that the wavefronts produced by the pressure contours inside
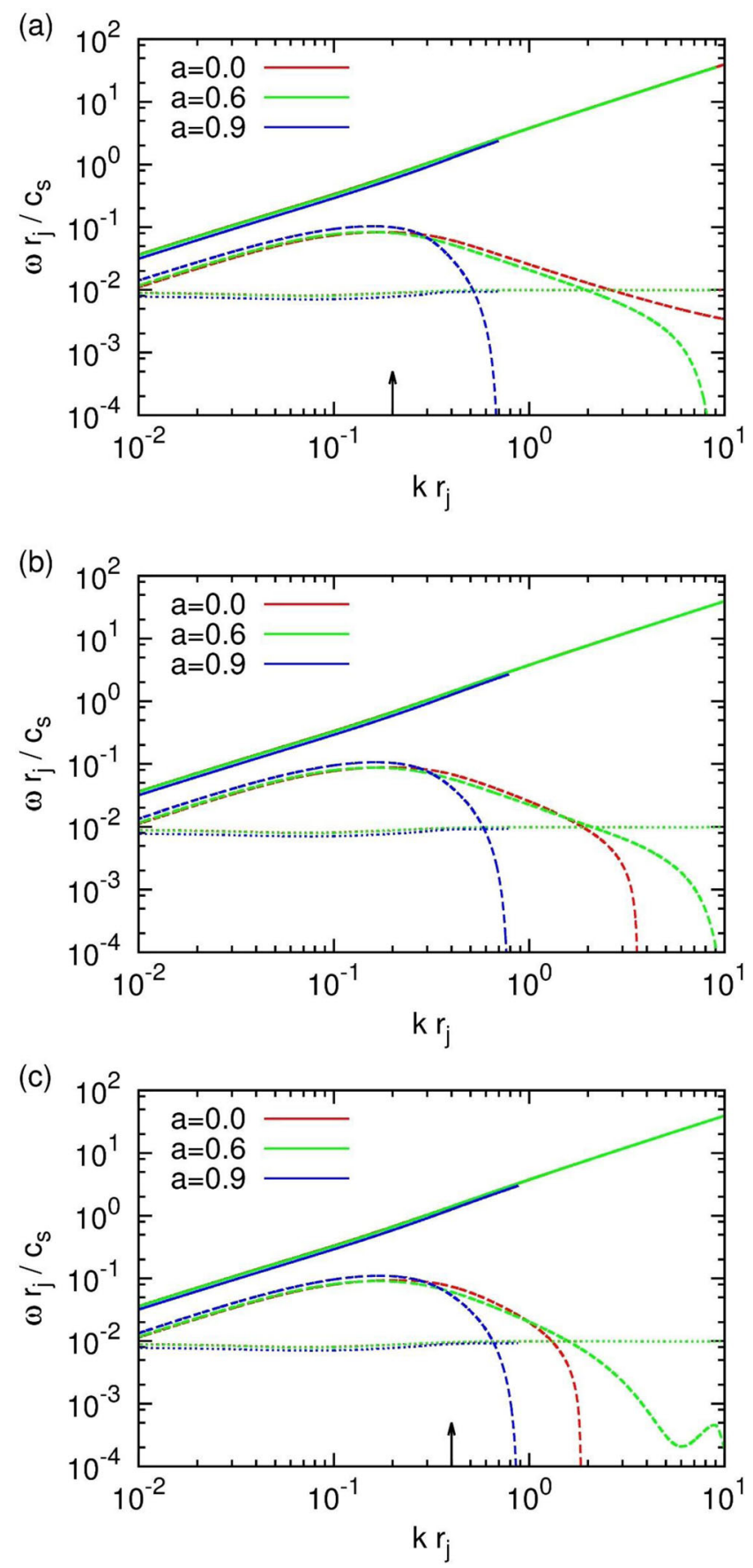

Figure 9. The angular frequency (solid line) and temporal growth rate (dashed line) as a function of longitudinal wavenumber $k$ for kink $(m=1)$ fundamental mode of (a) a non-magnetized jet, (b) a jet with $\beta=1$ (i.e. magnetic field is in equipartition with gas pressure) and (c) a jet with $\beta=0.5$ (magnetically dominated). The jet has $M=4$ and $\eta=0.1$. Increasing values of the parameter ' $a$ ' indicate increasing shear, with $a=0$ (no shear) to $a=0.9$ (maximal shear).

and outside the jets make the same angle w.r.t. the surface of the jet. Then please observe that this is not the case in Fig. 8c.) At large wavenumber, the propagation angle inside of the jet for the reflection mode asymptotically approaches 90 -degree and the modes die out (' $R$ ' becomes smaller). Please also see fig. 5 in Payne \& Cohn (1985). This implies that the fluctuation inside of the jet becomes longitudinal as the wavenumber increases. This perspective, which 

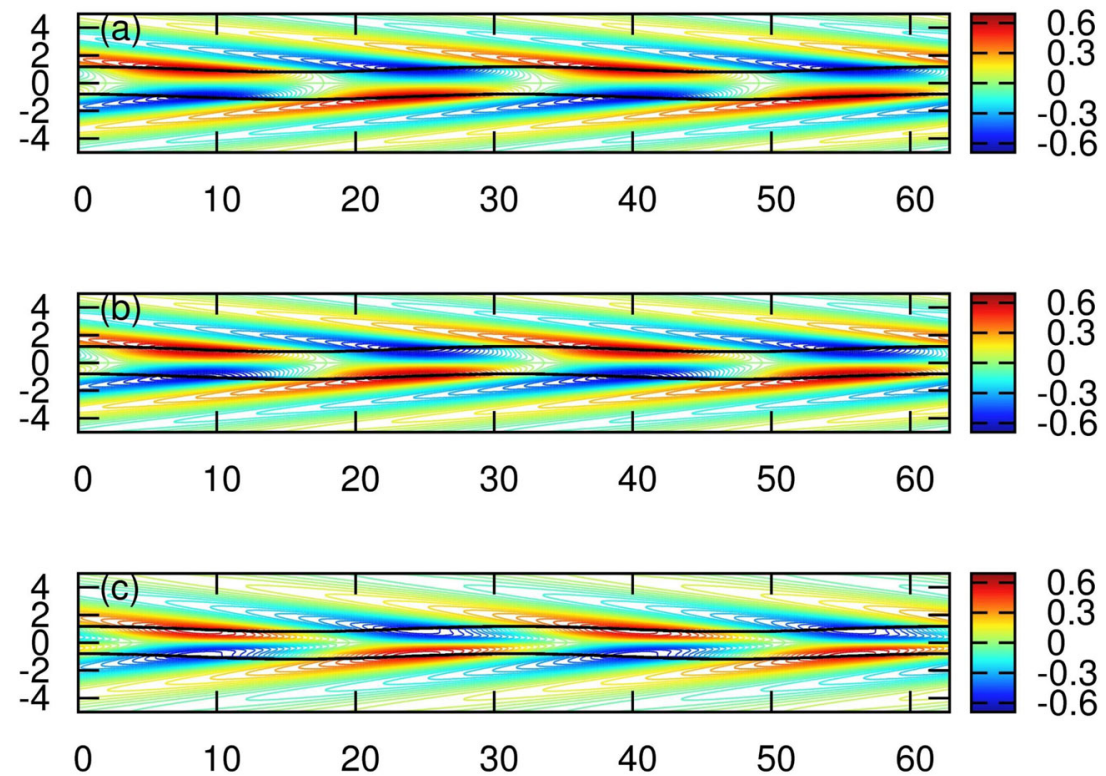

Figure 10. The pressure variation of the fundamental kink mode in a non-magnetized jet with (a) a top hat profile, (b) $a=0.6$ (moderate shear) and (c) $a=0.9$ (strong shear). For all the cases in Fig. 10 we have $k r_{j}=0.25$. In all the cases, the jet's boundary has a fluctuation that is 20 per cent of the jet's radius. The pressures are all on the same scale so that the pressures across panels within a figure can be inter-compared.
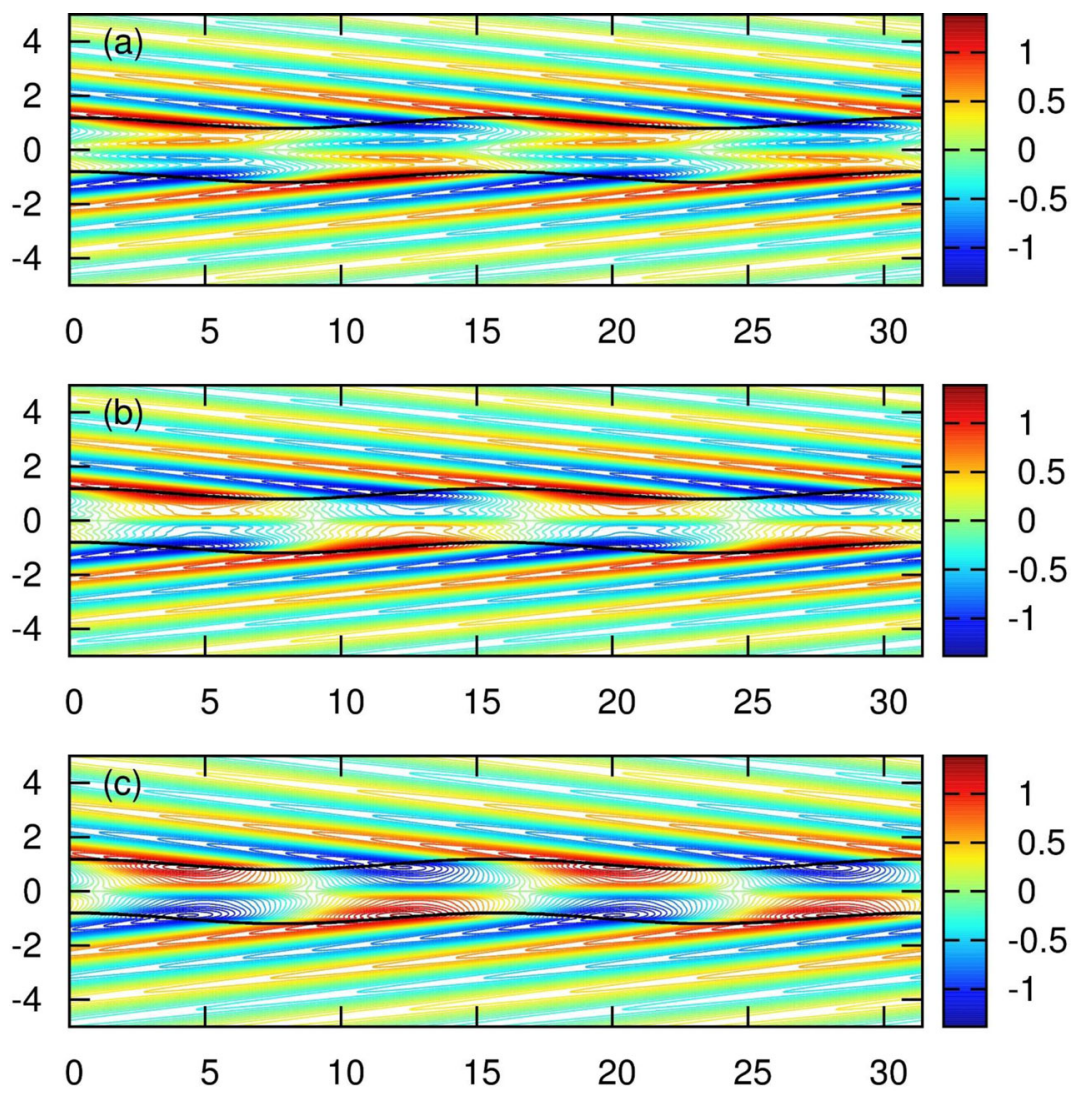

Figure 11. The pressure variation of the fundamental kink mode in a strongly magnetized jet ( $\beta=1 / 2$ ) with (a) a top hat profile, (b) $a=0.6$ (moderate shear) and (c) $a=0.9$ (strong shear). For all the cases in Fig. 11 we have $k r_{j}=0.25$. In all the cases, the jet's boundary has a fluctuation that is 20 per cent of the jet's radius. The pressures are all on the same scale so that the pressures across panels within a figure can be inter-compared.

is based on the reflection coefficient, is consistent with our physical interpretation that the velocity shear improves the stability of the jet. The concentrated pressure fluctuations close to the axis of the jet in Fig. 8(c) cause the wave that is propagating inside the jet be more longitudinal. On the other hand, the external propagation angle remains almost constant since the phase velocity does not depend much on the velocity shear (see solid line in Fig. 6). Therefore, the resulting reflection coefficient, ' $R$ ', for the sheared jet in 

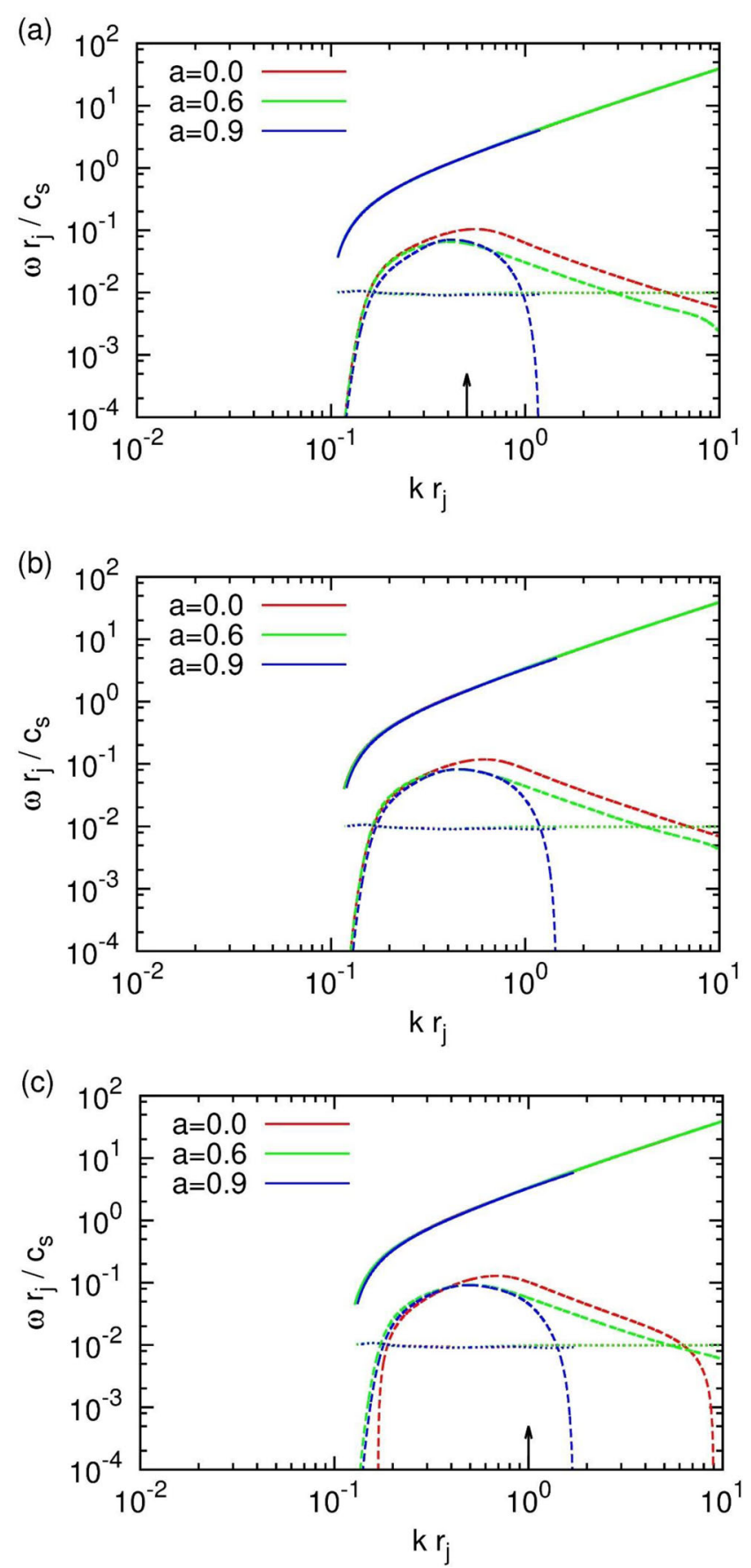

Figure 12. The angular frequency (solid line) and temporal growth rate (dashed line) as a function of longitudinal wavenumber $k$ for kink $(m=1)$ first reflection mode of (a) a non-magnetized jet, (b) a jet with $\beta=1$ (i.e. magnetic field is in equipartition with gas pressure) and (c) a jet with $\beta=0.5$ (magnetically dominated). The jet has $M=4$ and $\eta=0.1$. Increasing values of the parameter ' $a$ ' indicate increasing shear, with $a=0$ (no shear) to $a=0.9$ (maximal shear).

Fig. 8(c) has a smaller value compared with the value of ' $R$ ' for the jet without velocity shear in Fig. 8(a). Consequently, the velocity shear can enhance the stability of the jet in Fig. 8(c) compared to Figs 8(a) and (b).

\section{THE STABILITY OF THE FUNDAMENTAL MODE OF THE KINK INSTABILITY}

Fig. 9 shows dispersion relation analogous to Fig. 3 for the fundamental mode of the kink instability. Using our stability criteria, which are shown by the dotted lines in Fig. 9, increasing the shear in the velocity results in improved stability in the fundamental mode of the kink instability only at short wavelength. This fact is also valid for the magnetized jets in Figs 9(b) and (c).

The kink mode instabilities are thought to destabilize the jet and disrupt the jet structure easily. Furthermore, the strong magnetic field does not help to stabilize this mode since the strong magnetic field makes the current driven instability emerge from the jet and it becomes dominant in the kink mode instabilities. Many previous studies have shown special concern for the kink mode instabilities (Appl \& Camenzind 1992; Istomin \& Pariev 1994, 1996; Begelman 1998; Lyubarskii 1999; Tomimatsu et al. 2001; Narayan, Li \& Tchekhovskoy 2009; Bodo et al. 2013, 2016). This fact is observed in Fig. 9. Strong shear can stabilize the jet only at short wavelength, however, it cannot affect the stability at long wavelength. Even for the strongly magnetized jet, the velocity shear can only help to stabilize the jet at short wavelength (see Fig. 9c). The long wavelength unstable fundamental kink mode can only be stabilized by resorting to higher Lorentz factor (or higher Mach number) in the jet (Kim et al. 2017).

Fig. 10 shows pressure fluctuation for the unstable fundamental mode of the kink instability in a non-magnetized jet. The wavenumber that we choose is $k r_{\mathrm{j}}=0.2$ which is marked in Fig. 9(a) by an arrow. Figs 10(a)-(c) show the pressure perturbation for a nonmagnetized jet with $a=0,0.6$ and 0.9 , respectively. We again observe that the pressure fluctuation is concentrated on the jets' axis within the jet channel and much less concentrated at the boundary when the velocity shear increases. Please compare Fig. 10(a) with Fig. 10(c).

Fig. 11 is analogous to Fig. 10 but for the strongly magnetized ( $\beta=1 / 2$ ) jets. The wavenumber that we choose is $k r_{\mathrm{j}}=0.4$ which are marked in Fig. 9(c). Same trends are shown for the strongly magnetized jet, i.e. the fluctuation of pressure is confined at the axis of the jet and less concentrated on the surface.

\section{THE STABILITY OF THE FIRST REFLECTION MODE OF THE KINK INSTAB IL I T Y}

Fig. 12 shows dispersion relation analogous to Fig. 3 for the first reflection mode of the kink instability. Again, the velocity shear enhances the stability of the first reflection mode of the kink instability at short wavelength. This trend is valid even for the strongly magnetized jet. The stabilizing effect at short wavelength only appears when there is a velocity shear. In other words, there is no significant stabilizing effect due to the magnetic field at short wavelength for the first reflection mode of the kink instability.

Fig. 13 shows pressure fluctuation for the unstable first reflection mode of the kink instability in a non-magnetized jet. The wavenumber that we choose is $k r_{\mathrm{j}}=0.5$ which is marked in Fig. 12(a) by an arrow. We again observe that the pressure fluctuation is concentrated on the jets' axis within the jet channel while it is less concentrated at the boundary as the velocity shear increases. We choose the wave number that is very close to the wavenumber at resonance in Fig. 13(a). The propagation angle inside and outside jet at the jet's surface have almost same value at the wavenumber that we show in Fig. 13(a). 

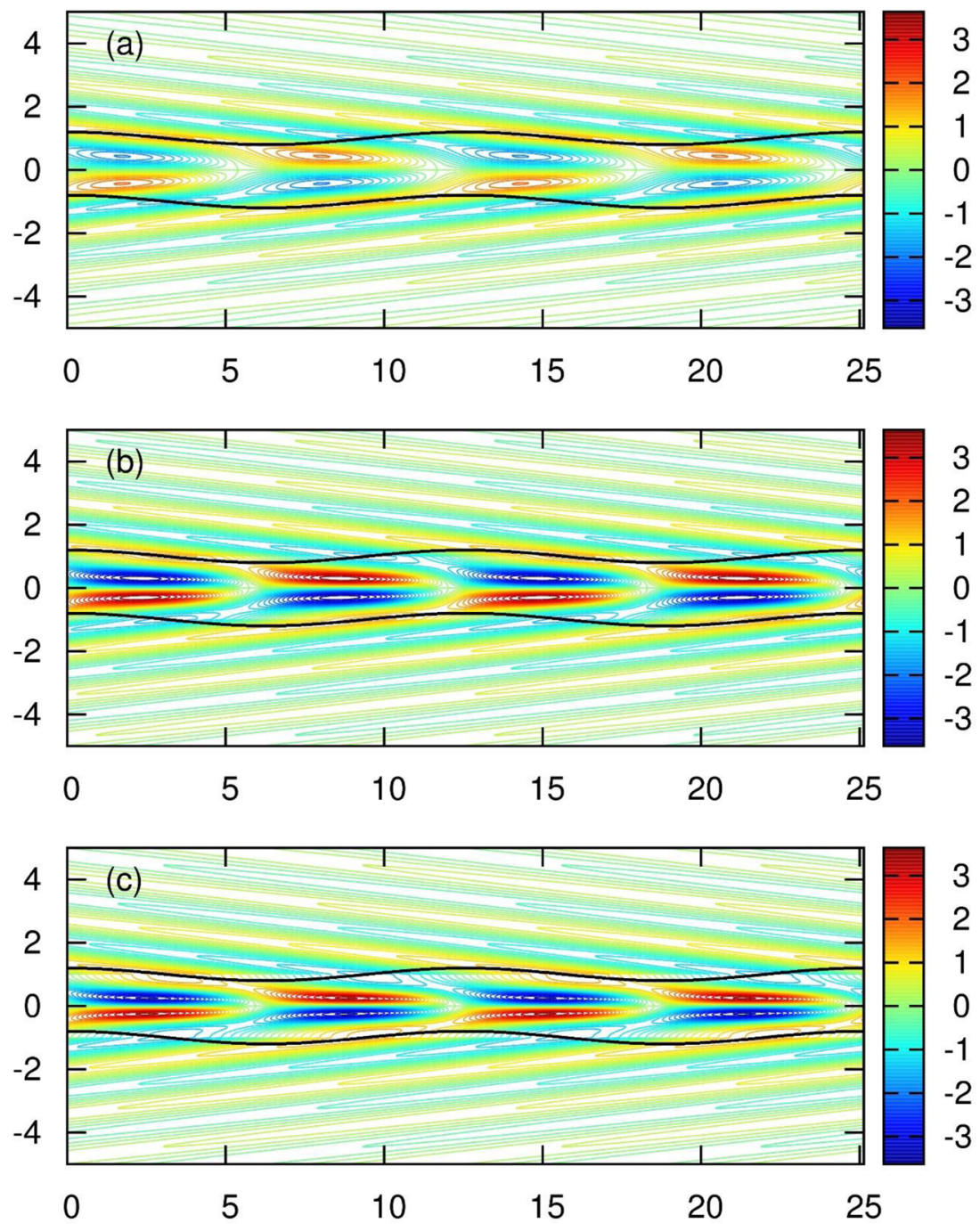

Figure 13. The pressure variation of the first reflection kink mode in a non-magnetized jet with (a) a top hat profile, (b) $a=0.6$ (moderate shear) and (c) $a=0.9$ (strong shear). For all the cases in Fig. 13 we have $k r_{j}=0.25$. In all the cases, the jet's boundary has a fluctuation that is 20 per cent of the jet's radius. The pressures are all on the same scale so that the pressures across panels within a figure can be inter-compared.

Fig. 14 is analogous to Fig. 13 but for strongly magnetized jets. The wavenumber that we choose is $k r_{\mathrm{j}}=1$ which is marked in Fig. 12(c) by an arrow. Again the pressure fluctuation is concentrated on the jets' axis within the jet channel. The propagation angles of the sound wave are almost similar values in Fig. 14(a). The difference between two angles can be observed in Fig. 14(c) since the wavenumber is away from the resonance.

\section{DISCUSSION AND CONCLUSIONS}

Astrophysical jets, such as AGN, GRB or protostellar jets, are observed to be incredibly stable. Keeping their integrity, they can propagate over 10 million times of their initial radii. It is one of the more remarkable facts of astrophysical jets compared to the terrestrial jets, which can extend only for few tens to hundreds of their radii.

In this series of studies, we used our numerical approach to study the linear stability of jets with non-trivial internal velocity, pressure and magnetic structure, which could not be handled using the traditional analytic approach. In order to ensure continuity with the previous investigations of jet stability, and to clearly identify the im- pact of various facets of jet structure on jet stability, we have been gradually progressing from simple basic models towards more realistic ones. Our studies of the stability of non-relativistic jets culminated in models with (1) strong velocity shear, (2) current-sheet free magnetic structure and (3) non-vanishing thermal pressure (Kim et al. 2015, 2016). Following in the steps of this non-relativistic study, our first paper on relativistic jets was dedicated to magnetic jets with a traditional top-hat velocity profile (Kim et al. 2017). In the current study of relativistic jets, we added a velocity shear inside the jet, yet allowing for a finite tangential discontinuity at the jet boundary.

For the choice of velocity shear, we use a parabolic profile of the spatial component of the 4-velocity instead of the 3 -velcity. This choice of velocity profile prevents an unphysical value of Lorentz factor near the axis of the jet. Thus, the jet 3-velocity profile is almost flat near the axis and falls off rapidly at the surface. The vortex sheet at the surface of the jet in this paper is stronger than that in the non-relativistic sheared jet. Accordingly, the unstable mode of the Kelvin-Helmholtz instability is less stabilized by the velocity shear. This is just an artefact of our parametrization of the velocity shear. (Unfortunately, there is no other reasonably tractable 

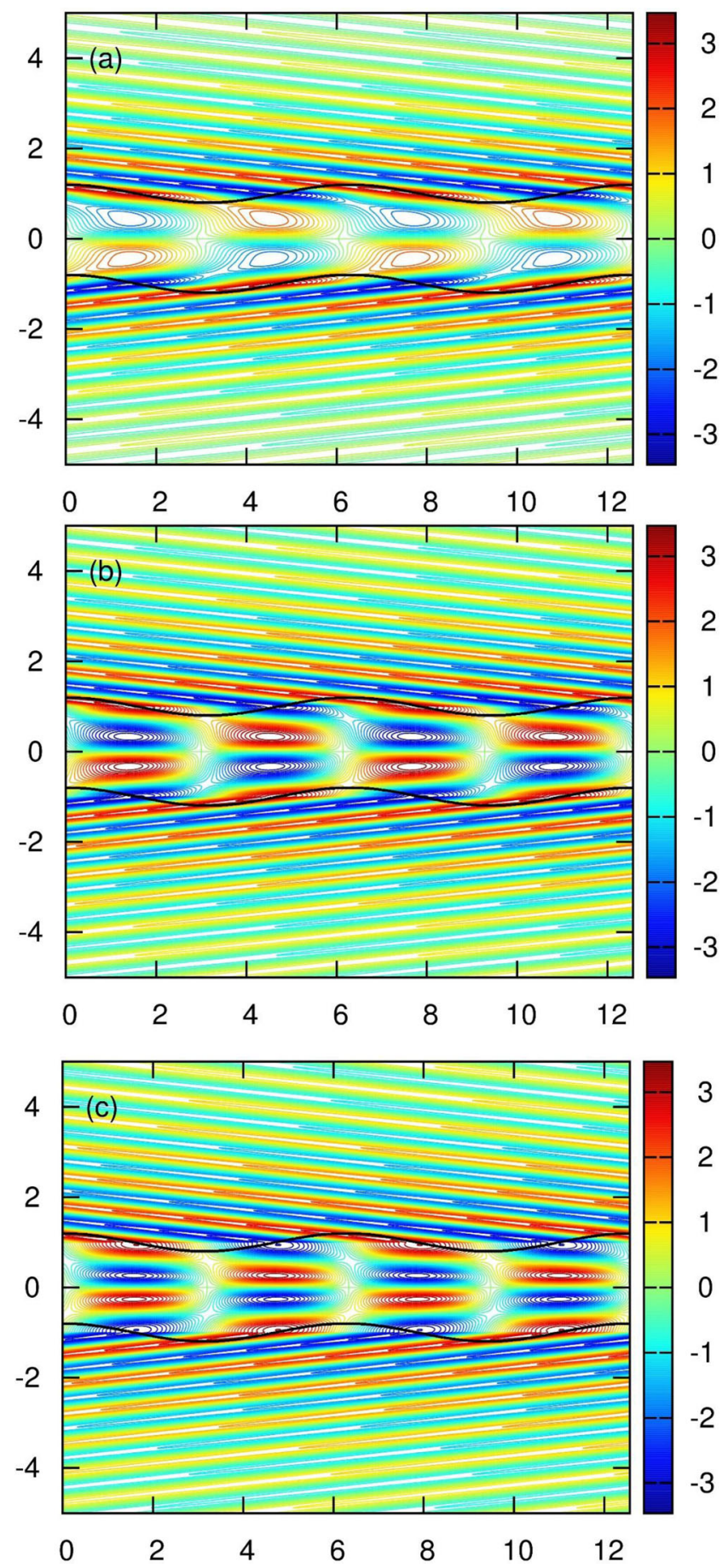

Figure 14. The pressure variation of the first reflection kink mode in a strongly magnetized jet ( $\beta=1 / 2)$ with (a) a top hat profile, (b) $a=0.6$ (moderate shear) and (c) $a=0.9$ (strong shear). For all the cases in Fig. 14 we have $k r_{\mathrm{j}}=0.2$. In all the cases, the jet's boundary has a fluctuation that is 20 per cent of the jet's radius. The pressures are all on the same scale so that the pressures across panels within a figure can be inter-compared.

parametrization that we can think of for relativistic jets that will not also engender superluminal motion in the jet.)

As expected from our prior non-relativistic stability analysis, inclusion of a velocity shear significantly enhances the stability of relativistic jets. The velocity shear mainly plays a role in stabilizing the short wavelength modes for all classes of unstable modes. In ad- dition, it also stabilizes the long wavelength fundamental modes of the pinch instability. Combining velocity shear with a strong magnetic field makes relativistic jets even more stable. Narayan, Li \& Tchekhovskoy (2009) claim that when the jet has the monotonically increasing or decreasing internal jet velocity (Lorentz factor) profile with increasing radius, the velocity shear makes jet stable. Whereas, the jet becomes unstable if there is a maximum or minimum velocity at a location inside jet. This fact is completely consistent with the Rayleigh criterion (Rayleigh 1896). Since we only use the monotonically decreasing velocity profile, our results completely agree with Narayan, Li \& Tchekhovskoy (2009) as well as Rayleigh (1896). Our visualization of the fluctuation in the total pressure enables us to physically interpret how the velocity shear enhances the stability of a relativistic jet. As the velocity shear increases, the fluctuation is concentrated near the axis of the jet and this makes the fluctuation die down gradually at the surface of the jet. As a result, only small amount of the beam energy of the jet is lost through the surface of the jet. We also explain this enhancement of stability using the reflection coefficient which is dependent on the propagation angle of the fluctuation.

This study is linear and does not include contributions from nonthermal sources of pressure. Therefore, we cannot quantify how bright the pressure blobs in Figs 4, 5, 9 and 10 might be. However, we can point out relative trends; especially if we make the reasonable assumption that the non-thermal pressure fluctuations track the thermal pressure fluctuations. From Figs 4(c) and 5(c) we realize that shear causes the thermal pressure fluctuations to be concentrated more along the axis of the jets. As a result, sheared jets might be more centre-brightened compared to their unsheared counterparts. Similarly, from Figs 9(c) and 10(c) we see an analogous trend in the fundamental modes of the kink instability. Comparing Fig. 8(c) to Figs 8(a) and (b) reveals a similar trend for the reflection modes of the pinch instability. Furthermore, comparing Fig. 14(c) to Figs 14(a) and (b) confirms an analogous trend for the reflection modes of the kink instability.

All our results in this paper contain the Kelvin-Helmholtz (KH) instability as well as current driven (CD) instability. In other words, it is not possible to isolate one specific instability from the other in our model of the jet. Bodo et al. (2013) use a different magnetic field profile from ours. Hence, they can show the effect of the $\mathrm{KH}$ instability only when they use $m=-1$ that does not form a resonant surface. Here, the resonant surface is a surface where the resonance condition $\left(k_{\mathrm{B}}=k B_{\mathrm{z} 0}+(m / r) B_{\phi 0}=0\right)$ holds. When the resonant surface is formed inside the jet, the CD instability starts to play a role to destabilize the jet. In our model, this surface always appears inside jet regardless the value of $k$ and $m$. As a result, the nonmagnetized or mildly magnetized (kinetically dominated) jets only show the KH instabilities while both $\mathrm{KH}$ and $\mathrm{CD}$ instabilities appear in the jets with high magnetization.

None of the models considered in our studies exhibits total suppression of instabilities though the range of length scales over which jets are unstable is significantly reduced because of the inclusion of realistic effects. Further stabilization might be obtained by permitting lateral expansion of the jet. This occurs when the jet propagates through a stratified external medium. A number of previous studies have pointed out that such an expansion could be the main reason behind the apparent suppression of global instabilities in astrophysical jets (e.g. Rosen \& Hardee 2000; Moll, Spruit \& Obergaulinger 2008; Porth \& Komissarov 2015). Our results provide an indirect support to this explanation.

Our results can be directly applicable to particular scales where the lateral expansion of astrophysical jets is small. For example, 
this may occur when an AGN jet enters its radio lobe and becomes re-collimated by the lobe's high gas pressure (e.g. Falle 1991; Komissarov \& Falle 1998). Weaker naked AGN jets may become re-collimated by the gas pressure of galactic X-ray corona (e.g. Porth \& Komissarov 2015). In fact, it is on these scales where according to the observations some AGN jets show the signs of developed instabilities. The process of re-collimation (and reconfinement of initially freely expanding supersonic jets) forces the jet plasma to flow along curved streamlines. This may create conditions favourable to development of the Rayleigh-Taylor-type instabilities (Matsumoto \& Masada 2013). Unfortunately, this potentially very important instability cannot be studied in the context of cylindrical models describing flows in a hydrostatic or magnetostatic equilibrium across the streamlines.

On another note, magnetic models of jet central engines predict that near their origin astrophysical jets should have strong rotational velocity. Further out, the rotation rate strongly decreases but near the central engine it can be high enough to have an impact on the jet stability. Recently, Bodo et al. (2016) studied the role of the rotation on the unstable mode of the magnetized jet in the Newtonian limit. We are planning to complete our studies of the linear stability by exploring the interplay between the magnetization and the rotation of the jet in the near future.

\section{ACKNOWLEDGEMENTS}

DSB acknowledges support via NSF grants NSF-DMS-1361197, NSF-ACI-1533850 and NSF-DMS-1622457. Several simulations were performed on a cluster at UND that is run by the Center for Research Computing. Computer support on NSF's XSEDE and Blue Waters computing resources is also acknowledged. SSK is supported via STFC grant ST/N000676/1.

\section{REFERENCES}

Appl S., 1996, A\&A, 314, 995

Appl S., Camenzind M., 1992, A\&A, 256, 354

Appl S., Lery T., Baty H., 2000, A\&A, 355, 818

Begelman M. C., 1998, ApJ, 493, 291

Birkinshaw M., 1991, MNRAS, 252, 505

Blandford R. D., 1976, MNRAS, 176, 465

Blandford R. D., Payne D. G., 1982, MNRAS, 199, 883

Blandford R. D., Znajek R. L., 1977, MNRAS, 179, 433

Bodo G., Rosner R., Ferrari A., Knobloch E., 1996, ApJ, 470, 797

Bodo G., Mamatsashvili G., Rossi P., Mignone A., 2013, MNRAS, 434, 3030

Bodo G., Mamatsashvili G., Rossi P., Mignone A., 2016, MNRAS, 462, 3031

Bonanno A., Urpin V., 2011, A\&A, 525, A100

Cohn H., 1983, ApJ, 269, 500

Falle S. A. E. G., 1991, MNRAS, 250, 581
Ferrari A., Massaglia S., Trussoni E., 1982, MNRAS, 198, 1065

Gourgouliatos K. N., Fendt C., Clausen-Brown E., Lyutikov M., 2012, MNRAS, 419, 3048

Hardee P. E., 1979, ApJ, 234, 47

Hardee P. E., 1982, ApJ, 257, 509

Hardee P. E., 2007, ApJ, 664, 26

Hardee P. E., Cooper M. A., Norman M. L., Stone J. M., 1992, ApJ, 399, 478

Hawley J. F., Krolik J. H., 2006, ApJ, 641, 103

Istomin Y. N., Pariev V. I., 1994, MNRAS, 267, 629

Istomin Y. N., Pariev V. I., 1996, MNRAS, 281, 1

Kim J., Balsara D. S., Lyutikov M., Komissarov S. S., George D., Siddireddy P. K., 2015, MNRAS, 450, 982

Kim J., Balsara D. S., Lyutikov M., Komissarov S. S., 2016, MNRAS, 461, 728

Kim J., Balsara D. S., Lyutikov M., Komissarov S. S., 2017, MNRAS, 467, 4647

Koide S., Meier D. L., Shibata K., Kudoh T., 2000, ApJ, 536, 668

Komissarov S. S., Falle S. A. E. G., 1998, MNRAS, 297, 1087

Komissarov S.S., 1999, MNRAS, 308, 1069

Komissarov S. S., Barkov M. V., 2009, MNRAS, 397, 1153

Komissarov S. S., Barkov M. V., Vlahakis N., Königl A., 2007, MNRAS, 380,51

Lister M. L. et al., 2009, AJ, 138, 1874

Lovelace R. V. E., 1976, Nature, 262, 649

Lyubarskii Y. E., 1999, MNRAS, 308, 1006

Matsumoto J., Masada Y., 2013, ApJ, 772, L1

McKinney J. C., 2006, MNRAS, 368, 1561

McKinney J. C., Blandford R. D., 2009, MNRAS, 394, L126

McKinney J. C., Narayan R., 2007, MNRAS, 375, 513

Moll R., Spruit H. C., Obergaulinger M., 2008, A\&A, 492, 621

Nalewajko K., Begelman M. C., 2012, MNRAS, 427, 2480

Narayan R., Li J., Tchekhovskoy A., 2009, ApJ, 697, 1681

Nishikawa K.-I., Richardson G., Koide S., Shibata K., Kudoh T., Hardee P., Fishman G. J., 2005, ApJ, 625, 60

Payne D. G., Cohn H., 1985, ApJ, 291, 655

Perucho M., Marti J. M., Hanasz M., 2005, A\&A, 443, 863

Perucho M., Hanasz M., Marti J. M., Miralles J. A., 2007, Phys. Rev. E, 75 056312

Piran T., 2004, Rev. Mod. Phys., 76, 1143

Porth O., Komissarov S. S., 2015, MNRAS, 452, 1089

Rayleigh L. W. S. B., 1896, The Theory of Sound, Vol. 2. Macmillan, London

Rees M. J., 1978, Nature, 275, 516

Reipurth B., Bally J., Fesen R. A., Devine D., 1998, Nature, 396, 343

Rosen A., Hardee P. E., 2000, ApJ, 542, 750

Rykoff E. S. et al., 2009, ApJ, 702, 489

Tchekhovskoy A., Narayan R., McKinney J. C., 2011, MNRAS, 418, 79L

Tomimatsu A., Matsuoka T., Takahashi M., 2001, Phys. Rev. D, 64, 123003

Urpin V., 2002, A\&A, 385, 14

This paper has been typeset from a $\mathrm{T}_{\mathrm{E}} \mathrm{X} / \mathrm{LAT}_{\mathrm{E}} \mathrm{X}$ file prepared by the author. 